interconnections, and a major player in Earth's climate system. This is grist to the scientific mill — let debate ensue.

Shanaka de Silva is in the Department of Space Studies, University of North Dakota, 526 Clifford Hall, Grand Forks, North Dakota 58202-9008, USA. e-mail:desilva@space.edu

1. Philander, S. G. H. El Niño, La Niña and the Southern Oscillation (Academic, San Diego, 1990).

2. Adams, J. B., Mann, M. E. \& Ammann, C. M. Nature 426, 274-278 (2003).

3. Hirono, M. J. Geophys. Res. 93, 5365-5384 (1988).

4. Robock, A. Rev. Geophys. 38, 191-219 (2000).

5. Handler, P. \& Andsager, K. Int. J. Clim. 10, 413-424 (1988).

\title{
Developmental biology
}

\section{Gender benders}

\author{
Peter Koopman
}

A painstaking triple-gene-knockout study has revealed a crucial role for insulin receptors in male sexual development. But is multiple-gene targeting the way forward for analysing genome function in mammals?

T he essential differences between the sexes seem constantly to perplex and beguile us. In mammals such as humans it's all down to the Y chromosome, of course: one either inherits it from one's father or one does not. Some 12 years ago, genetic studies identified what it is about this chromosome that, literally, makes a male. These studies culminated in the discovery of a Y-chromosomal gene known as Sry, and provided evidence that it is both necessary and sufficient for the development of testes ${ }^{1}$ - the first essential step in producing a male. But just how Sry works to bring about the cascade of gene activity leading to testis formation is still not understood. With the paper published on page 291 of this issue by Nef and colleagues ${ }^{2}$, we move a step closer to understanding the complexities of Sry and its role in triggering male development.

Nef et al. have produced mouse embryos lacking the function of all three members of the insulin-receptor family of genes. These genes are known as Ir (this encodes the insulin receptor), Igflr (encoding the receptor for insulin-like growth factor-1) and Irr (which produces the insulin-receptor-related receptor), and they are known to regulate various aspects of growth and metabolism. Several studies have suggested that these genes have overlapping functions that could be identified only by inactivating all three at once. Nef et al. have done just that. Their triple-gene-knockout embryos resulted from crosses between mice that had mutations in each of the genes individually, produced by experimental gene targeting in embryonic stem cells. None of the three single-knockout mouse strains had defects in sexual development. But the effect of the triple knockout was dramatic and surprising: the embryos with one $\mathrm{X}$ and one $\mathrm{Y}$ chromosome showed complete male-tofemale sex reversal. Their gonads resembled normal XX ovaries in appearance and in the expression of ovary-specific genes such as Wnt4 and Fig $\alpha$. But theylacked the expression of testis marker genes such as Sox9 and Amh.
What does this tell us about the role of the insulin receptors in sexual development? First, the sex reversal was observed very early in the development of the gonads, from the very first time at which it is usually possible to see the difference between testes and ovaries in mouse embryos. So the insulin receptors exert their influence early on.

More telling, perhaps, is that the sexreversed, triple-knockout embryos showed a dramatic reduction in the expression of Sry in the gonads, hinting that the three insulin receptors act upstream of this gene to control sex determination. Some Sry gene activity was retained, however, so the receptors are not absolutely required for Sry expression. It remains to be seen whether the lower expression levels result from reduced Sry activity in each gonadal cell, or whether fewer cells come to express Sry but produce it at the usual levels. Another question is how signalling from the three insulin receptors is transduced through gonadal cells to activate Sry expression, and how this signalling interacts biochemically and genetically with other factors implicated in Sry regulation; these include the product of the tumoursuppressor gene Wt1 (ref. 3), and the genetranscription factors GATA4 and FOG2 (ref.4).And, of course, the six-million-dollar question still eludes us: what happens after Sry is activated?

Nef et al. ${ }^{2}$ also found that gonadal cells in the triple-knockout embryos proliferated more slowly than their normal counterparts. This observation is significant because an increase in cell proliferation characterizes the early development of XY gonads compared with $\mathrm{XX}$ gonads ${ }^{5}$, and is thought to be important for boosting the population of cells that expresses testis-promoting factors, in order to outcompete the ovarian development pathway. So the finding that the insulin receptors are needed for rapid cell proliferation in the gonads supports the idea that the receptors are crucial to the early stages of sex determination. It is not yet clear, though, whether the insulin receptors affect cell proliferation in the gonads directly, as they do in other organs, or indirectly, via effects on Sry; the male-specific increase in proliferation is normally a consequence of Sryactivity ${ }^{5}$.

The study by Nef and colleagues reveals new and unexpected roles for the insulinreceptor family in sexual development, and goes some way to defining what those functions are. This adds to the known roles for insulin and insulin-like growth factor (Igf), signalling through Ir and Igf1r, in various metabolic and growth processes. In addition, it provides what may be the first known role for Irr, a receptor that is conserved between species but does not bind insulin or Igf molecules. An important goal of future research in sexual development will be to identify the molecule (or molecules) that does bind to Irr.

The effect of this triple-gene knockout was dramatic, and the experiment informative. Yet the logistics of generating mutant embryos that lack the function of three genes simultaneously are formidable. For instance, to inactivate a gene completely, both copies of it in the genome must be knocked out but mice lacking both copies of Ir alone die shortly after birth. The same happens with mice lacking Igflr alone. That meant that these mice couldn't be used for breeding to produce triple knockouts. So Nef et al. first generated mice that lacked one copy of each of these genes and both copies of Irr, and then used these animals for interbreeding to generate the triple-knockout embryos. Generating parents of this genotype would have been trouble enough, but the interbreeding would have produced XY triple-knockout embryos at a frequency of just 1 in 128.

It is clear that single-gene knockouts will become an inadequate tool for comprehensive analysis of gene function in mice, given that more and more gene families are being characterized in which different members act redundantly in the same biological process ${ }^{6}$. But it is also evident that generating multiple mutations by interbreeding of single-knockout animals will not be efficient on a large scale. Arguably, the future of functional gene analysis in complex genetic systems rests on the development of technologies such as RNA interference ${ }^{7}$, which should allow many genes to be disrupted simultaneously in vivo.

Peter Koopman is at the Institute for Molecular

Bioscience, University of Queensland, Brisbane,

Queensland 4072, Australia.

e-mail:p.koopman@imb.uq.edu.au

$$
\begin{aligned}
& \text { 1. Koopman, P., Gubbay, J., Vivian, N., Goodfellow, P. } \\
& \text { \& Lovell-Badge, R. Nature 351, 117-121 (1991). } \\
& \text { 2. Nef, S. et al. Nature 426, 291-295 (2003). } \\
& \text { 3. Hammes, A. et al. Cell 106, 651-659 (2001). } \\
& \text { 4. Tevosian, S. et al. Development 129, 4627-4634 (2002). } \\
& \text { 5. Schmahl, J., Eicher, E. M., Washburn, L. L. \& Capel, B. } \\
& \text { Development 127, 65-73 (2000). } \\
& \text { 6. Wellik, D. M. \& Capecchi, M. R. Science 301, 363-367 (2003). } \\
& \text { 7. Xia, H., Mao, Q., Paulson, H. L. \& Davidson, B. L. } \\
& \text { Nature Biotechnol. 20, 1006-1010 (2002). }
\end{aligned}
$$

